Amjad Kanj, MD, MPH

Division of Pulmonary and Critical

Care, Department of Medicine,

Mayo Clinic, Rochester, MN
Diane Levine, MD, FACP

Vice-chair for Education, and Professor, Department of Internal Medicine, Wayne State University, Detroit, MI

\title{
Overcoming obesity: Weight-loss drugs are underused
}

\begin{abstract}
A propose solutions.

\section{DIET AND EXERCISE FAIL FOR MOST} LTHOUGH WEIGHT-LOSS DRUGS are safe and effective, they are underused by healthcare providers in the United States. In the struggle against obesity, we need all the tools at our disposal, including prescription drugs. In this article, we examine barriers and

Obesity is a public health concern that has become a worldwide epidemic. An estimated two-thirds of US adults are either overweight

Successful management of obesity requires a multifaceted approach (body mass index $25-29.9 \mathrm{~kg} / \mathrm{m}^{2}$ ) or obese (body mass index $\geq 30 \mathrm{~kg} / \mathrm{m}^{2}$ ). ${ }^{1}$ Obesity is associated with a number of comorbidities such as hypertension, type 2 diabetes mellitus, cardiovascular diseases, obstructive sleep apnea, and infertility. ${ }^{2}$ Obesity-related healthcare expenditures in the United States amount to over $\$ 150$ billion annually. ${ }^{1}$

Lifestyle modifications continue to be the cornerstone of management, but most patients cannot achieve or maintain long-term meaningful weight loss by simply exercising or changing their diet. Pharmacotherapy is appropriate when lifestyle and behavioral interventions are ineffective and should be considered in all obese patients and in those with a body mass index $27 \mathrm{~kg} / \mathrm{m}^{2}$ or higher who have obesity-associated comorbidities. ${ }^{3}$

Until 2012, the lipase inhibitor orlistat was the only drug approved by the US Food and Drug Administration (FDA) for long-term treatment of obesity. Now there are 4 others: lorcaserin, phentermine and topiramate, naltrexone and bupropion, and liraglutide. ${ }^{3}$ These doi:10.3949/ccjm.87a.19102 drugs work by different mechanisms and result in a decrease of $5 \%$ to $10 \%$ in weight. ${ }^{1}$

In addition, antiobesity drugs are associated with reductions in high blood sugar, high blood pressure, low-density lipoprotein cholesterol, and severity of obstructive sleep apnea, and with improvement in cardiovascular and metabolic outcomes. ${ }^{1}$ These favorable effects, likely mediated by weight loss, make antiobesity prescription drugs an attractive option, especially considering that alternative options such as surgery are associated with a variety of additional risks.

\section{WHY DON'T PHYSICIANS PRESCRIBE ANTIOBESITY DRUGS?}

It is estimated that fewer than $2 \%$ of overweight and obese patients in the United States were ever prescribed antiobesity drugs. ${ }^{4}$ This may be owing to a variety of barriers.

\section{Physicians and patients are reluctant to talk about obesity}

Many physicians are not familiar with antiobesity prescription drugs or may lack the time to address weight loss with their patients. Some may avoid speaking about obesity during clinic visits due to the stigma associated with the topic. These factors are compounded by lack of appropriate reimbursement to these clinicians. ${ }^{5}$ This will result in missed opportunities for introducing antiobesity prescription drugs to the right patients.

Similarly, many patients avoid the topic of obesity because they feel ashamed, or they feel helpless to do anything about it or cannot afford to seek care for it. These factors are compounded by a lack of public health policies to prevent and counteract obesity in the United States. ${ }^{5}$ 
Obesity bias, negative attitudes toward individuals who are overweight and obese, is prevalent in the healthcare setting and represents a major barrier to medical care for these patients. This was highlighted in a systematic review by Puhl and Heuer ${ }^{6}$ that documented providers viewing their overweight and obese patients as "unmotivated," "noncompliant," "sloppy," and "lazy" and perceiving overweight as a behavioral problem caused by physical inactivity and food addiction. These negative attitudes were expressed not only by physicians and nurses but also by medical students, fitness professionals, and dietitians. Obesity bias has a substantial impact on healthcare utilization; obese patients who experience stigma are less likely to receive appropriate preventive care and counseling, and weight management. ${ }^{6}$

\section{Drugs have adverse effects}

Antiobesity prescription drugs are relatively safe but are not free of adverse effects that can affect tolerability. For instance, orlistat may cause bloating, diarrhea, and fecal incontinence, which tend to occur early in the treatment and subside as the patient learns to avoid fatty foods. Phentermine-topiramate is associated with dizziness, palpitations, and hand tremors owing to its sympathomimetic properties and may not be suitable for patients with arrhythmogenic heart diseases. ${ }^{2}$

Moreover, more than a dozen other drugs previously introduced for short- and longterm management of obesity were withdrawn by the FDA because of serious adverse effects. ${ }^{2}$ Fenfluramine and phentermine ("fen-phen") was withdrawn in 1997 because of its association with valvular heart disease. ${ }^{7}$ These events may have discouraged physicians from prescribing weight-loss drugs, and patients from taking them.

Another potential barrier to utilization of weight-loss drugs is adherence. Antiobesity drugs are often prescribed for a long duration, making adherence difficult for patients, especially if the desired weight-loss goal is not achieved. Furthermore, because of the modest effect of antiobesity drugs on weight, physicians may prescribe them at higher, offlabel dosages or prescribe non-FDA-approved drugs. Additionally, patients may resort to less expensive over-the-counter drugs, or drugs that have a more potent weight-loss effect but may also be addictive, such as amphetamines. ${ }^{8}$

\section{Insurance does not pay for them}

Gomez and Stanford ${ }^{9}$ found that only $11 \%$ of US health insurance plans offered coverage for antiobesity prescription drugs, in only 9 states. Medicaid had some form of coverage for antiobesity prescription drugs in only 8 states, and Medicare excluded all weight-loss drugs from part D coverage, even if prescribed for noncosmetic indications. This general lack of coverage may be partially due to a lack of studies of cost-effectiveness and of long-term costsavings associated with weight loss achieved by drugs. ${ }^{10}$

Recently, there have been efforts aimed at expanding Medicare part D coverage of FDAapproved antiobesity drugs. An example is the introduction of the Treat and Reduce Obesity Act of 2019, which also supports Medicare coverage for behavioral counseling for obesity by allowing other qualified healthcare providers such as advanced practice providers to offer these services. ${ }^{11}$

\section{PROPOSED SOLUTIONS}

The underuse of pharmacotherapy for obesity should be met by initiatives to educate patients on the available drugs, their benefits, and potential adverse effects.

At the same time, physicians and other prescribers should enhance their understanding of these drugs and thus gain confidence in prescribing them. This can be achieved with an early curricular focus on managing obesity and by setting up dedicated fellowships in obesity medicine for additional training.

Efforts should also be geared toward developing new drugs that are even more effective and have fewer adverse effects. Several antiobesity drugs are under investigation. ${ }^{1}$ As these new medications make their way from laboratory to pharmacy shelf, prescribers should be updated and encouraged to offer them to their patients.

Comprehensive health insurance coverage is also key. With the recognition of obesity as a disease, pharmacotherapies for it should be made affordable and accessible. Health policies are needed to lower the costs of weightloss drugs and expand health insurance cov- 
erage to include FDA-approved medications. Additionally, public awareness should be raised on the lack of efficacy data and potential adverse effects of non-FDA approved weight-loss drugs.

Successful management of obesity requires a multifaceted approach, starting with empathy stemming from the perception that obesity is a disease, not a stigma or series of bad choices. It is imperative that pharmacotherapy be used as an adjunct to lifestyle modifications and not as a substitute.
Physicians can assume an essential role, going beyond the treatment of obesity to address and manage underlying contributors, which include psychological stressors, socioeconomic barriers, dietary patterns, comorbidities, and drugs the patient is taking for those conditions. ${ }^{12}$ This demands detailed history-taking, regular follow-up visits, and a holistic and multidisciplinary approach, including intensive behavioral therapy and engagement of psychologists, nutritionists, and medical specialists when indicated. ${ }^{12}$

\section{REFERENCES}

1. Pilitsi E, Farr OM, Polyzos SA, et al. Pharmacotherapy of obesity: available medications and drugs under investigation. Metabolism 2019; 92:170-192. doi:10.1016/j.metabol.2018.10.010

2. Kang JG, Park CY. Anti-obesity drugs: a review about their effects and safety. Diabetes Metab J 2012; 36(1):13-25. doi:10.4093/dmj.2012.36.1.13

3. Kumar RB, Aronne $\mathrm{U}$. Pharmacologic treatment of obesity. In: Feingold KR, Anawalt B, Boyce A, et al, eds. Endotext. South Dartmouth, MA: MDText.com, Inc; 2000-2020.

4. Semla TP, Ruser C, Good CB, et al. Pharmacotherapy for weight management in the VHA. J Gen Intern Med 2017; 32(suppl 1):70-73. doi:10.1007/s11606-016-3949-y

5. Chan RS, Woo J. Prevention of overweight and obesity: how effective is the current public health approach. Int J Environ Res Public Health 2010; 7(3):765-783. doi:10.3390/ijerph7030765

6. Puhl RM, Heuer CA. The stigma of obesity: a review and update. Obesity (Silver Spring) 2009; 17(5):941-964. doi:10.1038/oby.2008.636

7. Onakpoya IJ, Heneghan CJ, Aronson JK. Post-marketing withdrawa of anti-obesity medicinal products because of adverse drug reactions: a systematic review. BMC Med 2016; 14(1):191. doi:10.1186/s12916-016-0735-y

8. Hendricks EJ. Off-label drugs for weight management. Diabetes Metab Syndr Obes 2017; 10:223-234. doi:10.2147/DMSO.S95299

9. Gomez G, Stanford FC. US health policy and prescription drug coverage of FDA-approved medications for the treatment of obesity. Int J Obes (Lond) 2018; 42(3):495-500. doi:10.1038/ijo.2017.287

10. Veerman JL, Barendregt JJ, Forster M, Vos T. Cost-effectiveness of pharmacotherapy to reduce obesity. PLoS One 2011; 6(10):e26051. doi:10.1371/journal.pone.0026051

11. US Congress. S.595-Treat and Reduce Obesity Act of 2019. Accessed July 30, 2020. https://www.congress.gov/bill/116th-congress/ senate-bill/595/text

12. Burguera B, Schauer $\mathbf{P}$, Kahan S. What to offer the $99 \%$ of patients with severe obesity who do not undergo bariatric surgery? Mayo Clin Proc 2019; 94(6):957-960. doi:10.1016/j.mayocp.2018.11.020

Address: Amjad Kanj, MD, Department of Pulmonary and Critical Care Medicine, Mayo Clinic, 200 1st St. SW, Rochester, MN 55904;

kanj.amjad@mayo.edu 